\title{
Surface Chemistry of Perovskite Oxynitride Photocatalysts: A Computational Perspective
}

\author{
Maria Bouria , Silviya Ninova ${ }^{a}$, Hassan Ouhbi ${ }^{\text {ab }}$, Nathalie Vonrütia, and Ulrich Aschauer ${ }^{\star a}$
}

\begin{abstract}
Perovskite oxynitrides are an established class of photocatalyst materials for water splitting. Previous computational studies have primarily focused on their bulk properties and have drawn relevant conclusions on their light absorption and charge transport properties. The actual catalytic conversions, however, occur on their surfaces and a detailed knowledge of the atomic-scale structure and processes on oxynitride surfaces is indispensable to further improve these materials. In this contribution, we summarize recent progress made in the understanding of perovskite oxynitride surfaces, highlight key processes that set these materials apart from their pure oxide counterparts and discuss challenges and possible future directions for research on oxynitrides.
\end{abstract}

Keywords: Oxynitride · Photoanode $\cdot$ Photocatalysis · Surface chemistry · Water splitting

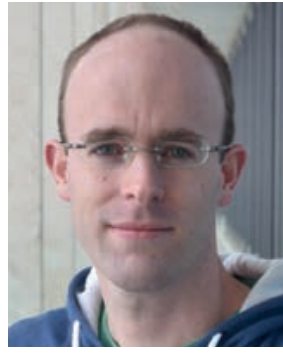

Ulrich Aschauer obtained his undergraduate and $\mathrm{PhD}$ degrees in material science and engineering at EPFL in 2005 and 2008 respectively, using classical atomistic simulation to investigate surface and interface properties in ceramic materials. During his postdoctoral appointments at Princeton University and ETH Zürich, he used density functional theory to investigate the effect of defects on the oxide surface chemistry and on functional properties of perovskite oxides respectively. Since 2016 he is an SNSF Assistant Professor at the Department of Chemistry and Biochemistry at the University of Bern, investigating oxynitride photocatalysts and defect-enabled functional oxides using density functional approaches.

\section{Introduction}

Hydrogen $\left(\mathrm{H}_{2}\right)$ is considered one of the most promising energy vectors for renewable energy and is presently part of future-energy strategies in many countries. The production of $\mathrm{H}_{2}$ from renewable sources proceeds mostly by two routes, photovoltaics coupled with water electrolysis or direct photocatalytic water splitting. ${ }^{[1]}$ While the former is based on mature technologies, the latter single-step conversion has the prospect of higher efficiencies, simpler and cheaper technology as well as reduced resource utilization. The realization of this promising approach is, however, still hampered by inefficiencies of the employed catalyst materials that do not drive the involved redox reactions sufficiently well. Of the two half-reactions involved in water splitting, the oxygen evolution reaction (OER)

$$
2 \mathrm{H}_{2} \mathrm{O}+4 \mathrm{~h}^{+} \rightarrow \mathrm{O}_{2}+4 \mathrm{H}^{+}
$$

is known to be kinetically more challenging than the hydrogen evolution reaction $\left(\right.$ HER) ${ }^{[2]}$

$$
4 \mathrm{H}^{+}+4 \mathrm{e}^{-} \rightarrow 2 \mathrm{H}_{2}
$$

Therefore, much research has been devoted to finding efficient OER catalysts, without which cost-effective water splitting is impossible. While in overall water-splitting setups, one catalyst drives both reactions, in the currently predominant photoelectrochemical (PEC) setups, the two reactions occur on different electrodes, and the photoanode material can be optimized for the OER alone. ${ }^{[3]}$ Traditionally semiconducting oxides such as $\mathrm{TiO}_{2}$ or $\mathrm{WO}_{3}$ were used for this purpose ${ }^{[4,5]}$ but it became apparent that such binary compounds cannot fulfill the requirements for an efficient photoanode, which are 1) a bandgap suitable to absorb a large part of the solar spectrum, 2) a valence band edge that provides holes with sufficient excess energy to drive the OER, while 3) also having high carrier mobilities and good exciton separation efficiencies, and 4) of course also catalyzing the targeted OER cycle with good efficiency. Binary oxides in particular suffer from too large band gaps - more than $3 \mathrm{eV}$ - which restricts light absorption to the small ultra-violet part of the solar spectrum, thus drastically reducing their conversion efficiencies. ${ }^{[6]}$ It was recognized that ternary, quaternary, or even more complex oxide materials show improved and more tunable properties, $\mathrm{BiVO}_{4}$ currently being one of the best performing oxides.[7] While oxides are known for their good chemical stability, it was found that compounds based on other anions, such as sulfides or nitrides can also have good properties for photoanode applications, while, however, generally having somewhat reduced chemical stabilities. Searching the vast chemical space of multinary oxides, nitrides and sulfides is an ongoing task, frequently guided by computational screening followed by experimental realization.

Apart from pure oxides, nitrides, or sulfides, mixed-anion photoanode materials such as oxysulfides and in particular oxynitrides have attracted tremendous interest in recent years. ${ }^{[8]}$ This is rooted in their properties that are somewhat intermediate between those of oxides and nitrides. They typically have improved chemical stabilities compared to pure nitrides but at the same time, due 
to the reduced electronegativity of nitrogen compared to oxygen, a smaller bandgap than pure oxides. Depending on their bandedge positions, this can make oxynitrides highly promising photoanode materials..$^{[9,10]}$ Oxynitrides can occur in different crystal structures with various fields of applications, for example as phosphors, ${ }^{[11]}$ pigments, ${ }^{[12]}$ and the notable case of tantalum oxynitride $(\mathrm{TaON})^{[13]}$ for applications in photocatalysis.

Oxynitrides can, however, also occur in the $\mathrm{ABX}_{3}$ perovskite crystal structure ${ }^{[14]}$ (Fig. 1a), where A and B are cations, while X is the anion ( $\mathrm{O}$ or $\mathrm{N})$. This crystal structure is famously known for its ability to accommodate a large number of different cationic elements, leading to a tremendous tunability of the resulting properties. The perovskite structure is also known to exhibit distortions such as octahedral rotations, B-cation off-centering, or Jahn-Teller distortions, that affect or even determine many of the functional properties. ${ }^{[15]}$ Oxygen-rich $\mathrm{ABO}_{2} \mathrm{~N}$ perovskite oxynitrides (Fig. 1b) are typically more active photocatalysts than their nitrogen-rich $\mathrm{ABON}$, counterparts ${ }^{[16]}$ (Fig. 1c). Considering only the $\mathrm{ABO}_{2} \mathrm{~N}$ class, the nominal anion oxidation states of $\mathrm{O}^{2-}$ and $\mathrm{N}^{3-}$ restrict the combinations of $\mathrm{A}$ and $\mathrm{B}$ cation oxidation states to add up to +7 , which is fulfilled with $\mathrm{A}^{2+}$ and $\mathrm{B}^{5+}$ as well as $\mathrm{A}^{3+}$ and $\mathrm{B}^{4+}$ pairs. In the former class, alkaline earth ions such as $\mathrm{Ca}^{2+}$, $\mathrm{Sr}^{2+}$ and $\mathrm{Ba}^{2+}$ can be paired with elements such as $\mathrm{Nb}^{5+}$ or $\mathrm{Ta}^{5+}$, while in the latter class lanthanides such as $\mathrm{La}^{3+}$ dominate, combined predominantly with first-row transition metals such as $\mathrm{Ti}^{4+}$. This results in the prototypical perovskite oxynitrides $\mathrm{SrTaO}_{2} \mathrm{~N}^{[17]}$ and $\mathrm{LaTiO}_{2} \mathrm{~N}^{[9]}$ that were previously synthesized and investigated as photocatalysts with bandgaps of around $2.1 \mathrm{eV}$ for both materials. ${ }^{[9,18]}$ In addition to the $\mathrm{ABOX}_{3}$ parent structure, derived layered crystal structures such as the Ruddlesden-Popper, ${ }^{[19,20]}$ Aurivillius, ${ }^{[21]}$ or Carpy-Galy ${ }^{[22]}$ phases lead to different structural motives and altered stoichiometries, with further potential to tune the properties of oxynitrides. a)

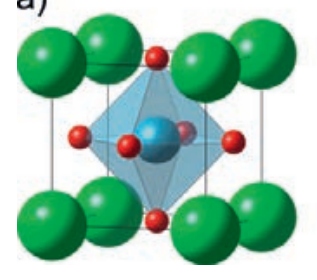

b)

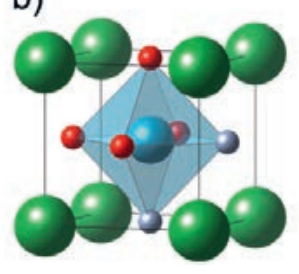

c)

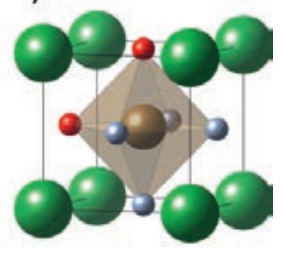

Fig. 1. a) Perfectly cubic $(P m \overline{3} m) \mathrm{ABO}_{3}$ perovskite oxide as well as the derived $b$ ) oxygen-rich $\mathrm{A}^{\prime} \mathrm{BO}_{2} \mathrm{~N}$ and c) nitrogen-rich $\mathrm{A}^{\prime} \mathrm{B}^{\prime} \mathrm{ON}_{2}$ oxynitrides. Color code: large green $=A$ (i.e. Sr), small green = A' (i.e. La), large blue $=\mathrm{B}$ (i.e. Ti), brown = B' (i.e. Ta), red = O, small blue $=\mathrm{N}$.

Compared to pure oxides, the mixed-anion nature of oxynitrides leads to an additional degree of freedom in how the two anions elements are arranged in the crystal structure. It is useful to make a distinction between short-range and long-range order. A short-range local cis anion order (Fig. 2a) is strongly favored in each $\mathrm{BO}_{4} \mathrm{~N}_{2}$ coordination octahedron compared to a trans arrangement (Fig. b) to optimize chemical bonding. On the other hand, only some perovskite oxynitride are known to display a partial $\left(\mathrm{SrNbO}_{2} \mathrm{~N}\right.$, $\left.\mathrm{SrTaO}_{2} \mathrm{~N}, \mathrm{BaTaO}_{2} \mathrm{~N}\right)$ or complete $\left(\mathrm{LaZrO}_{2} \mathrm{~N}\right)$ long-range anion order. This was rationalized by geometrically different but energetically similar cis anion orders in adjacent unit cells, leading to the absence of long-range order in most perovskite oxynitrides. This finding was supported by density functional theory (DFT) combined with cluster-expansion studies that report large energy differences in favor of cis anion orders but energetically very similar possibilities for different cis arrangements.[23,24] The anion order also affects the electronic structure, cis-ordered materials generally having somewhat larger bandgaps than trans-ordered structures.
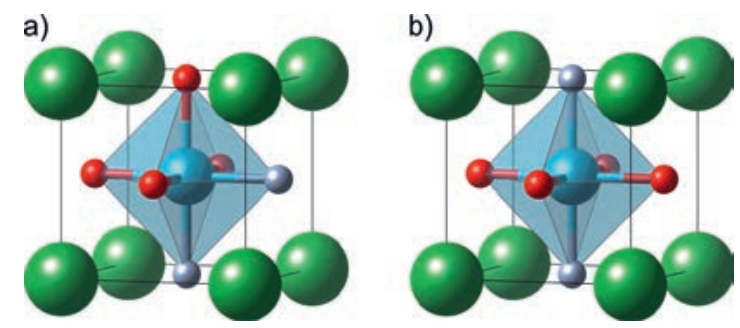

Fig. 2. $\mathrm{BO}_{4} \mathrm{~N}_{2}$ octahedron with a) cis order of the $\mathrm{N}$ anions and $\mathrm{b}$ ) trans order of the $\mathrm{N}$ anions. Color code: green = A (i.e. La), large blue = B (i.e. $\mathrm{Ti})$, red $=\mathrm{O}$, small blue $=\mathrm{N}$.

From the above, a generally very clear picture of the bulk structure and the anion order emerges, which can be used to justify the suitability of oxynitrides as photocatalysts due to their light absorption, exciton dissociation, and carrier mobility properties. These studies, however, neglect one crucial aspect of photocatalysis, which is the ionic and electronic structure of the surface that will dictate how the OER is catalyzed and what efficiencies can ultimately be reached.

\section{Surface Structure and Anion Order at Perovskite Oxynitride Surfaces}

Perovskite oxynitrides are typically synthesized by two approaches that will affect the orientation of the exposed surfaces. The first route starts from particles of a layered Carpy-Galy[22] oxide precursor $\left(\right.$ i.e. $\mathrm{La}_{2} \mathrm{Ti}_{2} \mathrm{O}_{7}$ ) that is placed under ammonia flow at high temperature for extended times. This process, called ammonolysis, induces an exchange of oxygen by nitrogen and ultimately the transformation of the layered to a normal perovskite structure. ${ }^{25]}$ The approach is believed to preserve the surface orientations exposed on the precursor, which are predominantly the pseudocubic (011) layering planes of the Carpy-Galy structure.[26] When, additionally, a flux is added, mass transport is enhanced and the oxynitride particles expose low-energy surface orientations according to their equilibrium morphology. ${ }^{[25]}$ For perovskite structured materials this is typically the pseudocubic (001) plane. [27] The other synthesis method is the preparation of thin films, typically grown by physical vapor deposition (PVD) such as pulsed laser deposition (PLD). Depending on the substrate and growth conditions, either epitaxial oriented ${ }^{[28-30]}$ or textured films ${ }^{[31]}$ can be obtained. The former expose the substrate surface, commonly (001), while the latter give rise to a collection of surfaces in proportions according to the texture, $\{001\},\{011\}$ and $\{112\}$ orientations being reported for $\mathrm{LaTiO}_{2} \mathrm{~N}$, for instance. ${ }^{[31]}$ The predominance of the (001) surface irrespective of the growth method, makes it the most relevant to study. Experimentally very little is known about the atomic-scale structure and composition of this particular orientation for perovskite oxynitrides; scanning transmission-electron microscopy (STEM) combined with electron energy-loss spectroscopy (EELS) shows a La termination for $\mathrm{LaTiO}_{2} \mathrm{~N}$, however only clearly observable after an aqua regia treatment that may have altered the surface. ${ }^{[32]}$

Computationally (001) surfaces were investigated for $\mathrm{CaTaO}_{2} \mathrm{~N}$ of varying bulk anion orders and for different facets of the $\{001\}$ family. ${ }^{[33]}$ The anion order was shown to largely affect the band gaps and band-edge positions, with some combinations of anion order and surface planes even being unable to drive the OER. ${ }^{[33]}$ In our work, we investigated both $\mathrm{LaTiO}_{2} \mathrm{~N}$ and $\mathrm{SrTaO}_{2} \mathrm{~N}$ (001) surfaces. ${ }^{[34,35]}$ We found that the surface anion order does not have to follow the bulk anion order. In fact, for $\mathrm{LaTiO}_{2} \mathrm{~N}$ the surface energies of the trans anion order are significantly lower than for the cis order that is strongly (by up to $0.3 \mathrm{eV}$ ) preferred in the bulk. ${ }^{[34]}$ This finding can be rationalized in terms of the electrostatic energy of the surface. In $\mathrm{LaTiO}_{2} \mathrm{~N}$, a trans anion order along the surface normal leads to alternating $\mathrm{LaN}$ and $\mathrm{TiO}_{2}$ lay- 
ers throughout the slab (Fig. 3a). A cis order, on the other hand, results in $\mathrm{La}$ and Ti layers containing mixtures of $\mathrm{O}$ and $\mathrm{N}$ anions (Fig. 3b). While the stacking sequences along the surface normal are hence formally charge neutral for the trans order, they are not for the cis order. Indeed, cis-ordered surfaces are classified as Tasker type III, ${ }^{[36]}$ being polar and having a diverging electrostatic energy. ${ }^{[37]}$ In a DFT calculation, these surfaces will spontaneously undergo electronic reconstruction (i.e. electron transfer from the bottom surface of a slab to the top layer or vice versa), which will generally lead to high surface energies. ${ }^{[38]}$ This implies that the anion order is one way for an oxynitride surface to compensate polarity. Compared to other possible compensation mechanisms, such as ionic reconstruction, anion reordering leads to the most stable $\mathrm{LaTiO}_{2} \mathrm{~N}$ (001) surfaces that will, if equilibrium can be reached, hence adopt a trans structure. ${ }^{[34]}$ a)

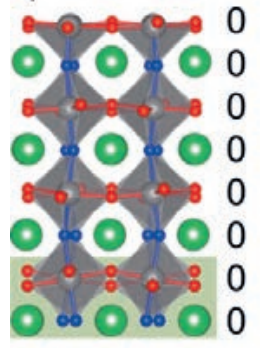

b)

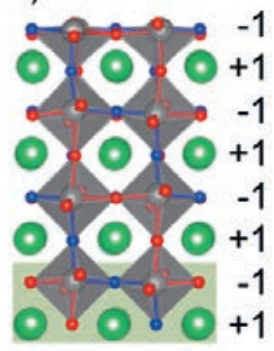

c)

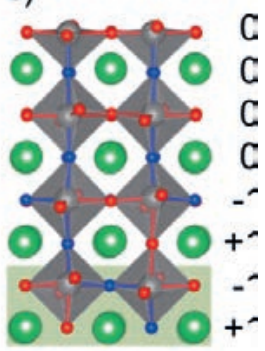

d)

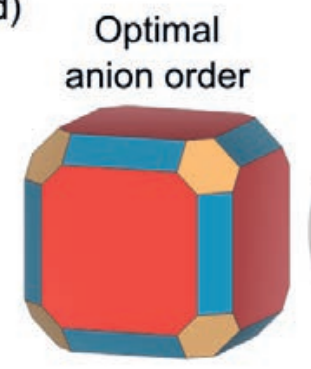

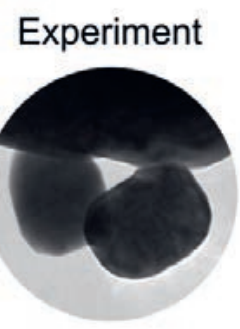

of anion reordering and ionic reconstructions to reach a state of low polarity that is then cancelled by small electronic reconstructions. By calculating the surface energies of a large set of differently reconstructed terminations with distinct anion orders for the experimentally motivated families of $\{011\},\{111\}$ and $\{113\}$ orientations, we have shown that the best agreement in equilibrium morphology between TEM experiment and DFT is obtained, when DFT models allow for a change in anion order towards trans ${ }^{[41]}$ (Fig. 3d). Anion reordering at oxynitride surfaces is hence a general phenomenon and an additional way for mixed-anion materials to reduce their surface energy compared to pure oxides or pure nitrides. This, however, also implies that the exact state of a surface is a complex interplay of ionic reconstructions, anion reordering, and small electronic reconstructions that is difficult to predict $a$ priori, generally requiring detailed calculations for each surface. Nevertheless, given the importance of the active-site coordination for the catalytic activity (vide infra), such knowledge is crucial for understanding the catalytic function of different oxynitrides.

The above discussion for $\mathrm{LaTiO}_{2} \mathrm{~N}$ has shown that a tendency for anion reordering exists at oxynitride surfaces. The question is if this a general feature of all oxynitrides or only some of them. By performing a similar set of calculations for $\mathrm{SrTaO}_{2} \mathrm{~N},{ }^{[35]}$ it was found that a cis bulk order also leads to charge neutral $\mathrm{SrO}$ and $\mathrm{TaON}$ layers along the (001) surface normal. The lowest energy (001) surfaces of tantalate oxynitrides, as well as niobate nitrides, are hence non-polar with the cis anion order that is most stable in the bulk and these materials will not exhibit the cis/trans interface found in $\mathrm{LaTiO}_{2} \mathrm{~N}$. On the other hand, assuming a catalytically active transition-metal terminated (001) surface, this implies that tantalate and niobate oxynitrides will expose $\mathrm{N}$ atoms at the surface, whereas for titanate oxynitrides, they are buried below the surface in the La layer, a difference in surface chemistry that will become important in sections 3 and 4.

a)
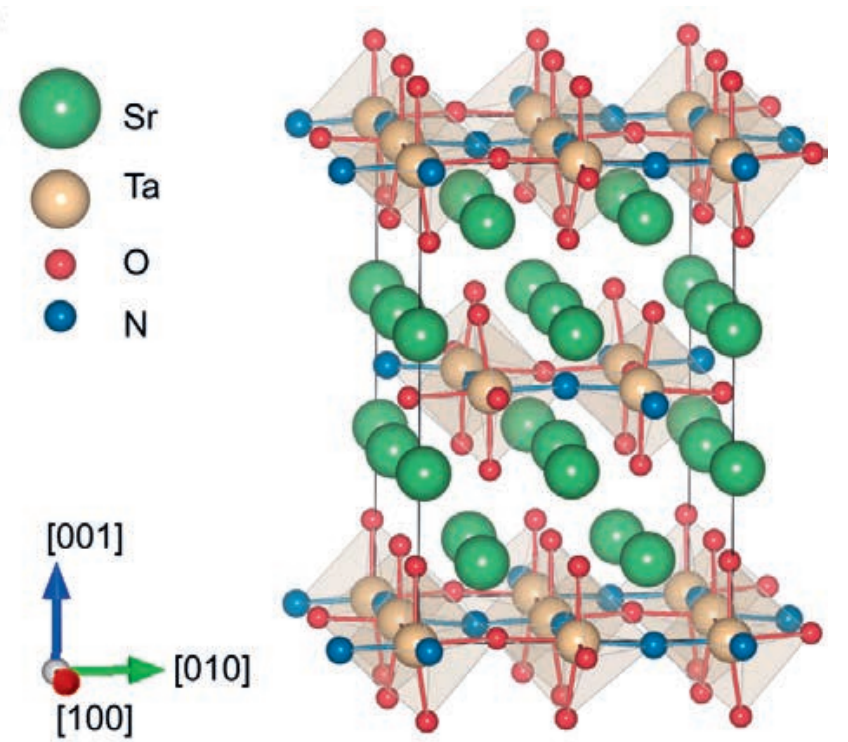

b) energy. The presence of the trans surface layer and the associated trans/cis interface seems to have a beneficial effect on photocatalysis, slightly lowering the predicted OER overpotential from 0.58 to 0.50 V. ${ }^{[39]}$ These values are slightly higher than the 0.37 $\mathrm{V}$ predicted by the universal scaling relations ${ }^{[40]}$ for the best performing catalyst but still indicate that $\mathrm{LaTiO}_{2} \mathrm{~N}$ is a fairly active OER catalyst.

While anion reordering was postulated as the energetically most favorable way to compensate polarity for the (001) orientation, surfaces along other Miller index planes may be polar independent of the anion order. These terminations may undergo a combination

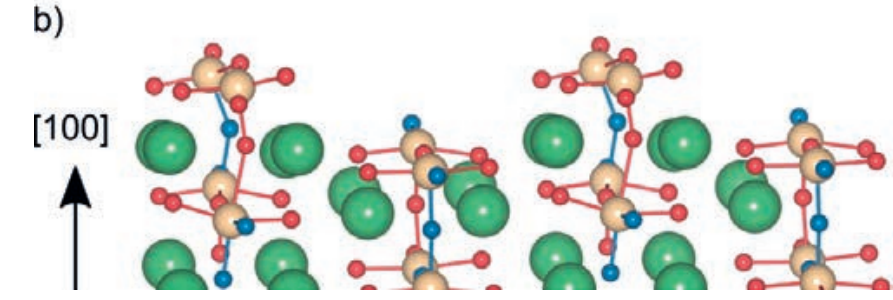

Fig. 4. a) $\mathrm{Sr}_{2} \mathrm{TaO}_{3} \mathrm{~N}$ Ruddlesden-Popper oxynitride bulk structure and b) the photocatalytically most relevant stepped (100) surface. Adapted with permission from ref. [43], copyright 2020 American Chemical Society. 
Turning finally to Ruddlesden-Popper structured oxynitrides and the composition $\mathrm{Sr}_{2} \mathrm{TaO}_{3} \mathrm{~N}^{[42]}$ (Fig. 4a), they expose, along the (001) direction, very similar surface structures as $\mathrm{SrTaO}_{2} \mathrm{~N}$. The layered structure, however, affects the binding energy of OER intermediate species and places $\mathrm{Sr}_{2} \mathrm{TaO}_{3} \mathrm{~N}$ on the weak-binding side of the OER activity volcano, while $\mathrm{SrTaO}_{2} \mathrm{~N}$ is located on the strong-binding side. ${ }^{[43]}$ This suggests that layering, such as in the Ruddlesden-Popper structure, can be a way to tune the reactivity of OER catalysts that bind reaction intermediates too strongly. The (001) orientation in the Ruddlesden-Popper structure, however, suffers from very low carrier mobility (very large effective masses) perpendicular to the surface. ${ }^{[42]}$ Therefore, other surface orientations, and in particular the (100) plane may be more relevant for PEC applications. We have shown that this orientation will leach $\mathrm{SrO}$ species and adopt a terraced surface structure (Fig. $4 \mathrm{~b}$ ), the Ta sites on the ridges having a fairly low OER overpotential of only $0.55 \mathrm{~V} \cdot{ }^{[43]}$ Moreover, as opposed to the (001) surface, only the (100) surface was shown to provide sufficiently energetic holes to drive the OER at its surface sites, further highlighting the relevance of this surface for PEC applications.

\section{Role of Nitrogen in Oxynitride Surface Chemistry}

Perovskite oxynitrides may expose $\mathrm{N}$ atoms at their surface. While this is not the case for the transition-metal terminated surfaces of $\mathrm{LaTiO}_{2} \mathrm{~N}(001)$ when the equilibrium anion order can be reached, ${ }^{[34]}$ it will be the case for tantalate oxynitrides, ${ }^{[35]}$ also in Ruddlesden-Popper form ${ }^{[42]}$ as well as orientations other than (001) for titanate oxynitrides. ${ }^{[41]}$ Under OER conditions (with an applied bias, under illumination, or a combination thereof) these oxynitride surfaces will, according to computed surface Pourbaix diagrams, be covered with a layer of $\mathrm{O}$ adsorbate molecules. In our studies, we observed the spontaneous association of these $\mathrm{O}$ adsorbates with surface $\mathrm{N}$ atoms, forming $\mathrm{O}-\mathrm{N}$ dimer species. ${ }^{[35,43]}$ According to the computed electronic structure, these dimers carry less negative charge than the separate ionic $\mathrm{O}^{2-}$ and $\mathrm{N}^{3-}$ spe-
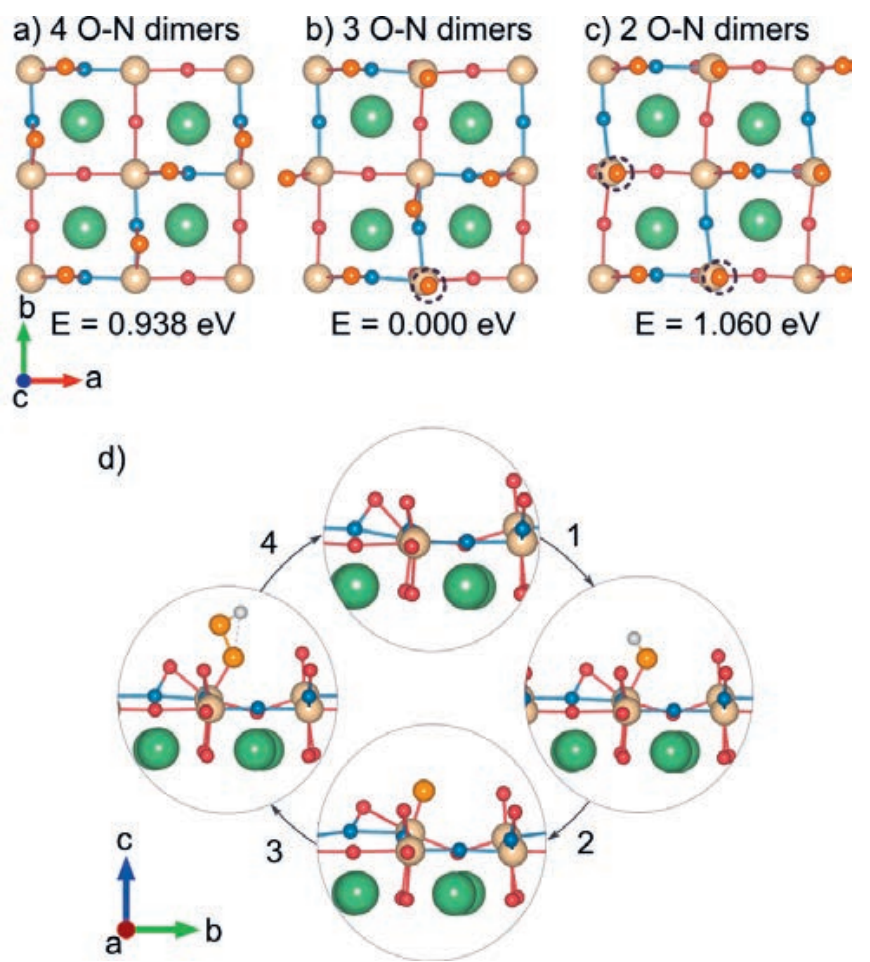

Fig. 5. a)-c) top view of configurations with 4, 3, and 2 O-N dimers on the $\mathrm{Sr}_{2} \mathrm{TaO}_{3} \mathrm{~N}$ (001) surface along with the relative energy. Panel d) shows how the OER can occur on a nominally overcoordinated Ta next to an $\mathrm{O}-\mathrm{N}$ dimer on $\mathrm{Sr}_{2} \mathrm{TaO}_{3} \mathrm{~N}$ (001). Adapted with permission from ref. [43], copyright 2020 American Chemical Society. cies. ${ }^{[43]}$ Dimer formation thus reduces the degree of oxidation the surface undergoes under OER conditions and therefore strongly stabilizes the surface adsorbate structure. In our calculations, we however observed not a complete conversion of all $\mathrm{O}$ adsorbates to $\mathrm{O}-\mathrm{N}$ dimers, but rather about $75 \%{ }^{[43]}$ (compare Figs $5 \mathrm{a}-\mathrm{c}$ ). This finding was rationalized by the fact that occupied states associated with the O-N dimer lie higher in energy than those associated with the upright $\mathrm{O}$ adsorbate and that there is hence a balance between the energy gained by $\mathrm{O}-\mathrm{N}$ formation and the accommodation of electrons is higher-energy states.

The formation of O-N dimers will, however, have a marked effect on the surface chemistry. The OER is commonly assumed to follow the following sequence of steps, where $*$ designates an active (transition-metal) site on the surface and $\mathrm{h}^{+}$is a hole:[44,45]

$$
\begin{array}{ll}
*+\mathrm{H}_{2} \mathrm{O}+\mathrm{h}^{+} \rightarrow * \mathrm{OH}+\mathrm{H}^{+} & (* \mathrm{OH} \text { formation }) \\
* \mathrm{OH}+\mathrm{h}^{+} \rightarrow * \mathrm{O}+\mathrm{H}^{+} & (* \mathrm{O} \text { formation }) \\
* \mathrm{O}+\mathrm{H}_{2} \mathrm{O}+\mathrm{h}^{+} \rightarrow * \mathrm{OOH}+\mathrm{H}^{+} & (* \mathrm{OOH} \text { formation }) \\
* \mathrm{OOH}+\mathrm{h}^{+} \rightarrow *+\mathrm{O}_{2}+\mathrm{H}^{+} & \left(\mathrm{O}_{2} \text { formation }\right)
\end{array}
$$

Since forming a dimer significantly lowers the energy of the $\mathrm{O}$ adsorbates and hence also $* \mathrm{O}$ OER reaction intermediates, it leads to a decrease in the free-energy change of the step to form the $* O$ intermediate. Since, however, the free-energy differences associated with formation of the $* \mathrm{O}$ and $* \mathrm{OOH}$ intermediates are constrained via the universal scaling relations to sum to $3.2 \mathrm{eV},{ }^{[40]}$ this will lead to an increase in the free-energy change of the $* \mathrm{OOH}$ formation step, typically resulting in a higher overpotential and hence a reduced catalytic activity of the oxynitride. These considerations could explain why titanate oxynitrides without surface $\mathrm{N}$ species are generall more active OER catalysts than tantalum oxynitrides with surface $\mathrm{N}$ species.

O-N dimers, even if spontaneously formed, may not necessarily have to participate in the OER as the OER could, for example, occur predominantly on the $\mathrm{O}$ adsorbates that remain upright. Apart from this possibility, we discovered for Ruddlesden-Popper $\mathrm{Sr}_{2} \mathrm{TaO}_{3} \mathrm{~N}$ that Ta atoms that carry an $\mathrm{O}-\mathrm{N}$ dimer and are hence formally fully coordinated, may become overcoordinated and catalyze the OER next to the adsorbed O-N dimer with a fairly small overpotential of only $0.51 \mathrm{~V}[43]$ (Fig. $5 d$ ). Since this was not observed for $\mathrm{SrTaO}_{2} \mathrm{~N}^{[35]}$ it is possible that the generally weaker binding of the Ruddlesden-Popper surface enables this alternative mechanism.

\section{Surface Defects and their Effect on Catalysis}

The reduced chemical stability of oxynitrides compared to oxides is expected to lead to a significant concentration of defects and in particular nitrogen vacancies due to the large energy gain by formation of the strong N-N triple bond in $\mathrm{N}_{2}$. Under OER conditions, characterized by a high oxygen and low nitrogen partial pressure, the formation of nitrogen vacancies is expected to be further enhanced compared to oxygen vacancies. When performing DFT calculations for $\mathrm{SrTaO}_{2} \mathrm{~N}$ with surface $\mathrm{N}$ vacancies under OER conditions (with surface $* \mathrm{O}$ adsorbates), we observed spontaneous healing of nitrogen vacancies by these oxygen adsorbates $^{[46]}$ (Fig. 6a). Such a process, on one hand, changes the surface stoichiometry and reduces the surface. On the other hand, though, it induces tensile strain into the surface as Ta-O bonds are shorter than Ta-N bonds but the surface is constrained to remain at the larger subsurface lattice parameters. This expansion of the surface structure leads to more facile $\mathrm{Ta}^{5+} \rightarrow \mathrm{Ta}^{4+}$ reduction and hence a preferential electron accommodation at the surface (Fig. $6 b)$. The excess electrons are expected to affect the photocatalytic 
properties in two ways: they will increase the chance of electronhole recombination in the surface region, but at the same time also directly affect the catalytic activity by significantly increasing the overpotential (Fig. 6c). The loss of surface nitrogen and its substitution with oxygen could hence be at the origin of the significant drop in current typically observed in the early stages of chronoamperometric measurements of oxynitride photocatalysts. ${ }^{[47,48]}$

Defect formation may, however, not necessarily be detrimental to the catalytic performance. This was previously reported in the so-called activity-stability relations ${ }^{[49-52]}$ that found less stable catalysts to generally be more active. Oxynitride photocatalysts tend to be operated under alkaline conditions where the materials are more stable. If operated under more neutral or even acidic conditions, the materials are expected to dissolve. This is a great challenge for theory as it implies that the OER will be catalyzed on an ever-changing surface structure and a single static surface, as usually used in DFT calculations, cannot correctly account for the observed catalytic activity. This motivated us to investigate the OER on a large variety of surfaces with different anion and cation defect configurations that hence expose a large variety of activesite geometries. ${ }^{[53]}$ Somewhat surprisingly, such a structural disorder brought about by dissolution leads to a large spread in catalytic activities compared to a single overpotential predicted for the stoichiometric and perfectly flat surface, even if thermodynamic and kinetic arguments are used to filter out irrelevant structures (Fig. 6d). The predicted overpotentials encompass a large range of the OER activity volcano, including sites at its very tip that represents the highest possible catalytic activity. Since turnover rates will depend exponentially on the overpotential, the apparent catalytic activity is expected to be dominated by a small number of such highly active sites, which provides a mechanistic explanation of the activity-stability relations.

\section{Challenges and Future Directions}

The computational research summarized above has led to a significant advance in our understanding of the surface structure and chemistry of mixed-anion oxynitride photocatalyst materials. In particular, it has revealed some aspects of these materials that may currently limit exploiting their full potential in PEC applications.

The above results show that $\mathrm{O}-\mathrm{N}$ dimer formation is prevalent on $\mathrm{B}^{5+}$ oxynitride surfaces and may significantly impact the free-energy profile of the OER. Moreover, $\mathrm{N}$ atoms present at the surface may be substituted by $\mathrm{O}$ during operation, leading to a reduced catalytic activity. Preventing O-N dimer formation and nitrogen loss would thus be beneficial for the catalytic activity but will be very difficult to achieve under the oxidizing conditions inherent to the OER. Here the different bonding in layered oxynitrides that enables alternative reaction mechanisms despite $\mathrm{O}-\mathrm{N}$ dimer formation could be one way to enhance reactivity. Another possibility that, however, increases the catalyst complexity would be to protect the oxynitride by a thin, catalytically highly active perovskite oxide layer that acts as the catalyst, while the oxynitride acts as the light absorber. Ideal candidate materials would, for example, be metallic perovskite oxides such as $\mathrm{SrRuO}_{3}$. Protective layers based on oxides or other oxynitrides (TaON) are established in oxynitride PEC engineering but the electronic structure of the combined oxide/oxynitride system and its suitability to not affect light absorption while enabling good charge transfer to the surface sites has not been investigated.

Protective layers as well as co-catalysts may also help to solve the long-term stability problem of oxynitrides. Hole accumulation at the surface may enable photocorrosive nitrogen dissolution, leading to a degradation of the electrodes. Preventing such processes by hole transfer to energetically more favorable states on a corrosion-resistant protection layer or co-catalyst could improve the long-term stability. A deeper understanding of interfaces be-
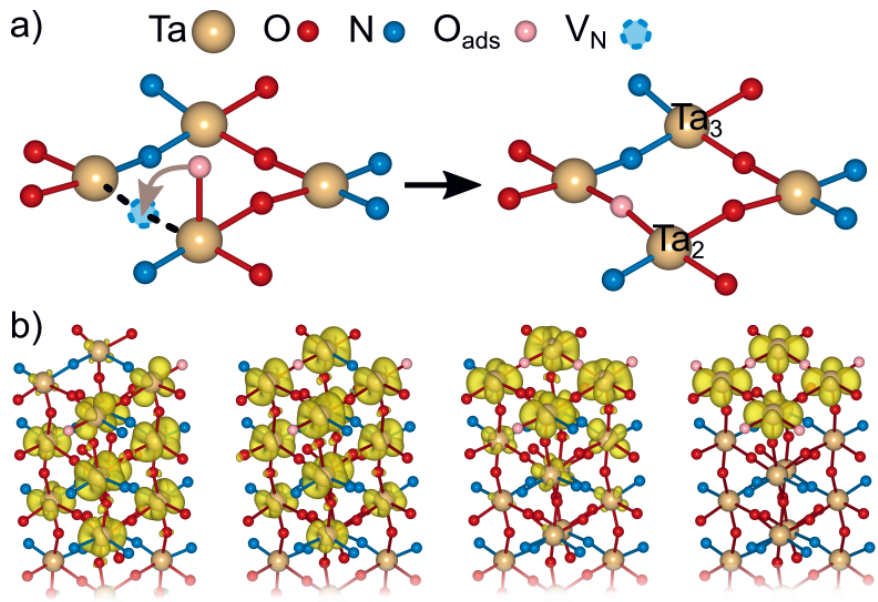

1 sub. $\mathrm{N}$

2 sub. $\mathrm{N}$

3 sub. $\mathrm{N}$

4 sub. $\mathrm{N}$
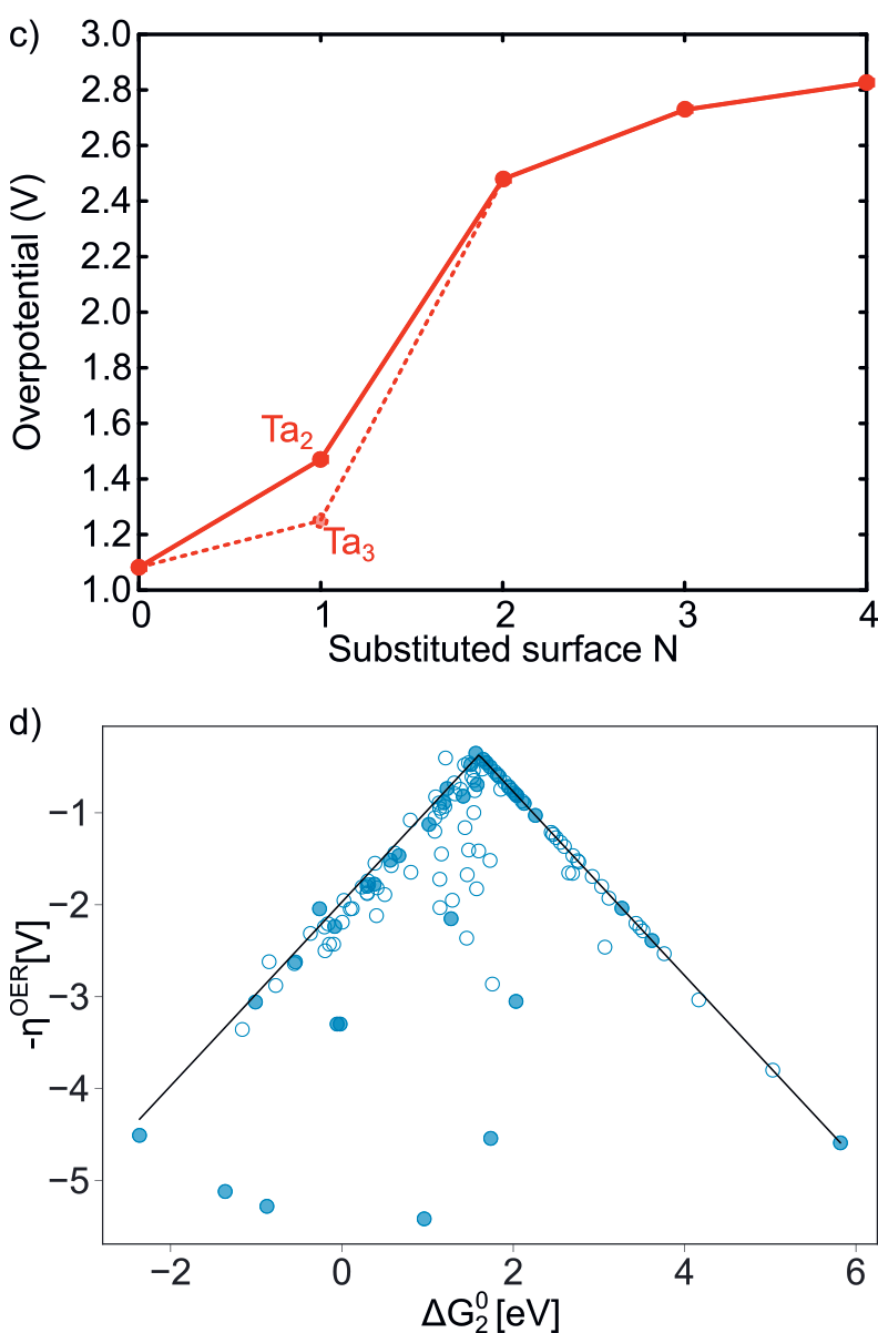

Fig. 6. a) Spontaneous healing of nitrogen vacancies $\left(V_{N}\right)$ by oxygen adsorbates $\left(\mathrm{O}_{\text {ads }}\right)$ on $\mathrm{SrTaO}_{2} \mathrm{~N}(001)$, b) excess electron density as a function of the number of substituted surface $\mathrm{N}$, showing the progressive electron localization at the surface, $c$ ) effect of substituting $\mathrm{N}$ by $\mathrm{O}$ on the OER overpotential, d) OER overpotential for different defective surface configurations on $\mathrm{LaTiO}_{2} \mathrm{~N}(001)$. The filled symbols show datapoints that are judged as relevant based on thermodynamic and kinetic considerations. Panels a-c adapted with permission from ref. [46], copyright 2019 American Chemical Society, panel d) adapted from ref. [53] published by The Royal Society of Chemistry.

tween oxynitrides and coating or co-catalyst materials will therefore be one of the requirements to further improve oxynitrides in PEC applications. 
Having understood the structure and chemistry of mixed-anion materials is, however, also the foundation to investigate even more complex multinary mixed-anion/mixed cation materials. ${ }^{[54]}$ Alloying different cations, either on the A or B site could alter the anion arrangement at the surface, potentially leading to $\mathrm{N}$-free surfaces like in $\mathrm{LaTiO}_{2} \mathrm{~N}$ that do not suffer from $\mathrm{O}-\mathrm{N}$ dimer formation or nitrogen loss. The presence of different cations is however expected to lead to complex strain fields and possibly surface segregation effects that need to be understood in detail to establish the surface geometry and reactivity of such materials and screen for ideal cation combinations.

\section{Acknowledgements}

We are grateful to Simone Pokrant, Sophia Haussener, Thomas Lippert, Daniele Pergolesi, Yasushi Hirose, and Tetsuya Hasegawa for highly enriching discussions and collaborations. This work was supported by the Swiss National Science Foundation Professorship grants PP00P2 157615 and PP00P2_187185. Calculations were performed on UBELIX (http://www.id.unibe.ch/hpc), the HPC cluster at the University of Bern, Piz Daint at CSCS under projects s676, s766, s955, and s1033 as well as SuperMUC at GCS@LRZ, Germany, for which we acknowledge PRACE for awarding us access.

Received: November 27, 2020

[1] Z. Wang, R. R. Roberts, G. F. Naterer, K. S. Gabriel, Int. J. Hydrogen Energ. 2012, 37, 16287, https://doi.org/10.1016/j.ijhydene.2012.03.057.

[2] H. Dau, C. Limberg, T. Reier, M. Risch, S. Roggan, P. Strasser, ChemCatChem 2010, 2, 724, https://doi.org/10.1002/cctc.201000126.

[3] H. Wu, H. L. Tan, C. Y. Toe, J. Scott, L. Wang, R. Amal, Y. H. Ng, Adv. Mater. 2020, 32, 1904717, https://doi.org/10.1002/adma.201904717.

[4] A. Fujishima, K. Honda, Nature 1972, 238, 37.

[5] C. Santato, M. Odziemkowski, M. Ulmann, J. Augustynski, J. Am. Chem. Soc. 2001, 123, 10639, https://doi.org/10.1021/ja011315x.

[6] M. D. Hernández-Alonso, F. Fresno, S. Suárez, J. M. Coronado, Energ. Environ. Sci. 2009, 2, 1231, https://doi.org/10.1039/b907933e.

[7] T. W. Kim, K.-S. Choi, Science 2014, 343, 990, https://doi.org/10.1126/science.1246913.

[8] H. Kageyama, K. Hayashi, K. Maeda, J. P. Attfield, Z. Hiroi, J. M. Rondinelli, K. R. Poeppelmeier, Nat. Commun. 2018, 9, 772, https://doi.org/10.1038/s41467-018-02838-4.

[9] A. Kasahara, K. Nukumizu, G. Hitoki, T. Takata, J. N. Kondo, M. Hara, H. Kobayashi, K. Domen, J. Phys. Chem. A 2002, 106, 6750, https://doi.org/10.1021/jp025961.

[10] A. E. Maegli, S. Pokrant, T. Hisatomi, M. Trottmann, K. Domen, A. Weidenkaff, J. Phys. Chem. C 2013, 118, 16344, https://doi.org/10.1021/jp4084162.

[11] T. Takeda, R.-J. Xie, T. Suehiro, N. Hirosaki, Prog. Solid State Chem. 2018, 51, 41, https://doi.org/10.1016/j.progsolidstchem.2017.11.002.

[12] R. Aguiar, D. Logvinovich, A. Weidenkaff, A. Rachel, A. Reller, S. G. Ebbinghaus, Dyes Pigments 2008, 76, 70, https://doi.org/10.1016/j.dyepig.2006.08.029.

[13] M. de Respinis, M. Fravventura, F. F. Abdi, H. Schreuders, T. J. Savenije, W. A. Smith, B. Dam, R. van de Krol, Chem. Mater. 2015, 27, 7091, https://doi.org/10.1021/acs.chemmater.5b02938.

[14] A. Fuertes, J. Mater. Chem. 2012, 22, 3293, https://doi.org/10.1039/c2jm13182j.

[15] J. M. Rondinelli, N. A. Spaldin, Adv. Mater. 2011, 23, 3363, https://doi.org/10.1002/adma.201101152.

[16] S. G. Ebbinghaus, H.-P. Abicht, R. Dronskowski, T. Müller, A. Reller, A. Weidenkaff, Prog. Solid State Chem. 2009, 37, 173, https://doi.org/10.1016/j.progsolidstchem.2009.11.003.

[17] E. Günther, R. Hagenmayer, M. Jansen, Zeits. Anorg. Allg. Chem. 2000, 626, 1519, https://doi.org/10.1002/1521-3749(200007)626:7<1519::aidzaac1519>3.0.co;2-i.

[18] X. Sun, F. Wu, G. Liu, X. Xu, J. Mater. Chem. A 2018, 6, 20760, https://doi.org/10.1039/c8ta06934d.

[19] S. N. Ruddlesden, P. Popper, Acta Crystallogr. 1957, 10, 538, https://doi.org/10.1107/s0365110x57001929.

[20] S. N. Ruddlesden, P. Popper, Acta Crystallogr. 1958, 11, 54, https://doi.org/10.1107/s0365110x58000128.

[21] B. Aurivillius, Arkiv foer Kemi 1949, 1, 463.

[22] M. N. Valdez, N. A. Spaldin, Polyhedron 2019, 171, 181, https://doi.org/10.1016/j.poly.2019.07.018.

[23] X. Xu, H. Jiang, J. Mater. Chem. A 2019, 7, 14583, https://doi.org/10.1039/c9ta01690b.

[24] X. Xu, H. Jiang, RSC Adv. 2020, 10, 24410, https://doi.org/10.1039/d0ra03681a.
[25] S. Pokrant, M. C. Cheynet, S. Irsen, A. E. Maegli, R. Erni, J. Phys. Chem. C 2014, 118, 20940, https://doi.org/10.1021/jp506597h.

[26] S. Pokrant, S. Dilger, S. Landsmann, J. Mater. Res. 2016, 31, 1574, https://doi.org/10.1557/jmr.2016.9.

[27] R. I. Eglitis, Ferroelectrics 2015, 483, 53 , https://doi.org/10.1080/00150193.2015.1058673.

[28] D. Oka, Y. Hirose, T. Fukumura, T. Hasegawa, Cryst. Growth Des. 2013, 14, 87, https://doi.org/10.1021/cg401176j.

[29] D. Oka, Y. Hirose, H. Kamisaka, T. Fukumura, K. Sasa, S. Ishii, H. Matsuzaki, Y. Sato, Y. Ikuhara, T. Hasegawa, Sci. Rep. 2014, 4, 4987, https://doi.org/10.1038/srep04987.

[30] D. Oka, Y. Hirose, F. Matsui, H. Kamisaka, T. Oguchi, N. Maejima, H. Nishikawa, T. Muro, K. Hayashi, T. Hasegawa, ACS Nano 2017, 11, 3860 , https://doi.org/10.1021/acsnano.7b00144.

[31] M. Pichler, W. Si, F. Haydous, H. Téllez, J. Druce, E. Fabbri, M. E. Kazzi, M. Döbeli, S. Ninova, U. Aschauer, A. Wokaun, D. Pergolesi, T. Lippert, $A d v$. Funct. Mater. 2017, 27, 1605690, https://doi.org/10.1002/adfm.201605690.

[32] M. Matsukawa, R. Ishikawa, T. Hisatomi, Y. Moriya, N. Shibata, J. Kubota, Y. Ikuhara, K. Domen, Nano Lett. 2014, 14, 1038, https://doi.org/10.1021/nl404688h.

[33] A. Kubo, G. Giorgi, K. Yamashita, Chem. Mater. 2017, 29, 539, https://doi.org/10.1021/acs.chemmater.6b03366.

[34] S. Ninova, U. Aschauer, J. Mater. Chem. A 2017, 5, 11040, https://doi.org/10.1039/c9ta01690b.

[35] H. Ouhbi, U. Aschauer, Surf. Sci. 2018, 677, 258, https://doi.org/10.1016/j.susc.2018.07.013.

[36] P. W. Tasker, J. Phys. C Solid State Phys. 2001, 12, 4977, https://doi.org/10.1088/0022-3719/12/22/036.

[37] C. Noguera, J. Phys.: Cond. Matter 2000, 12, R367.

[38] J. Goniakowski, C. Noguera, J. Phys. Condens. Matter 2014, 26, 485010, https://doi.org/10.1088/0953-8984/26/48/485010.

[39] S. Ninova, U. Aschauer, J. Mater. Chem. A 2019, 7, 2129 , https://doi.org/10.1039/c8ta10230a.

[40] I. C. Man, H.-Y. Su, F. Calle-Vallejo, H. A. Hansen, J. I. Martínez, N. G. Inoglu, J. Kitchin, T. F. Jaramillo, J. K. Nørskov, J. Rossmeisl, ChemCatChem 2011, 3, 1159, https://doi.org/10.1002/cctc.201000397.

[41] S. Ninova, S. Pokrant, U. Aschauer, ACS Appl. Energy Mater. 2020, 3, 5867 , https://doi.org/10.1021/acsaem.0c00777.

[42] M. Bouri, U. Aschauer, Phys. Chem. Chem. Phys. 2018, 20, 2771, https://doi.org/10.1039/c7cp06791g.

[43] M. Bouri, U. Aschauer, Chem. Mater. 2019, 32, 75, https://doi.org/10.1021/acs.chemmater.9b02294.

[44] J. Rossmeisl, A. Logadottir, J. K. Nørskov, Chem. Phys. 2005, 319, 178, https://doi.org/10.1016/j.chemphys.2005.05.038.

[45] J. Rossmeisl, Z. W. Qu, H. Zhu, G. J. Kroes, J. K. Nørskov, J. Electroanal. Chem. 2007, 607, 83, https://doi.org/10.1016/j.jelechem.2006.11.008.

[46] H. Ouhbi, U. Aschauer, ACS Mater. Lett. 2019, 1, 52, https://doi.org/10.1021/acsmaterialslett.9b00088.

[47] J. Seo, M. Nakabayashi, T. Hisatomi, N. Shibata, T. Minegishi, K. Domen, ACS Appl. Energy Mater. 2019, 2, 5777, https://doi.org/10.1021/acsaem.9b00908.

[48] J. J. Brown, Z. Ke, T. Ma, A. J. Page, ChemNanoMat 2020, 6, 708, https://doi.org/10.1002/cnma.201900703.

[49] S. H. Chang, J. G. Connell, N. Danilovic, R. Subbaraman, K.-C. Chang, V. R. Stamenkovic, N. M. Markovic, Faraday Discuss. 2014, 176, 125 https://doi.org/10.1039/c4fd00134f.

[50] N. Danilovic, R. Subbaraman, K.-C. Chang, S. H. Chang, Y. J. Kang, J. Snyder, A. P. Paulikas, D. Strmcnik, Y.-T. Kim, D. Myers, V. R. Stamenkovic, N. M. Markovic, J. Phys. Chem. Lett. 2014, 5, 2474 , https://doi.org/10.1021/jz501061n.

[51] S. H. Chang, N. Danilovic, K.-C. Chang, R. Subbaraman, A. P. Paulikas, D. D. Fong, M. J. Highland, P. M. Baldo, V. R. Stamenkovic, J. W. Freeland, J. A. Eastman, N. M. Markovic, Nat. Commun. 2014, 5, 4191, https://doi.org/10.1038/ncomms5191.

[52] C. Roy, R. R. Rao, K. A. Stoerzinger, J. Hwang, J. Rossmeisl, I. Chorkendorff, Y. Shao-Horn, I. E. L. Stephens, ACS Energy Lett. 2018, 3, 2045, https://doi.org/10.1021/acsenergylett.8b01178.

[53] N. Vonrüti, U. Aschauer, Phys. Chem. Chem. Phys. 2019, 21, 24354, https://doi.org/10.1039/c9cp04859f.

[54] S. Pokrant, A. E. Maegli, G. L. Chiarello, A. Weidenkaff, CHIMIA 2013, 67, 162, https://doi.org/10.2533/chimia.2013.162.

\section{License and Terms}

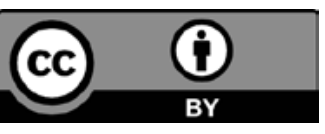

This is an Open Access article under the terms of the Creative Commons Attribution License CC BY 4.0. The material may not be used for commercial purposes.

The license is subject to the CHIMIA terms and conditions: (http:// chimia.ch/component/sppagebuilder/?view=page \&id=12).

The definitive version of this article is the electronic one that can be found at https://doi.org/10.2533/chimia.2021.202 